Pesq. Vet. Bras. 36(1):19-23, janeiro 2016 DOI: $10.1590 / \mathrm{S} 0100-736 \mathrm{X} 2016000100003$

\title{
Morte súbita em bovinos no Sul do Rio Grande do Sul: epidemiologia e diagnóstico ${ }^{1}$
}

\author{
Pablo Estima-Silva ${ }^{2}$, Kayane Rosales Molarinho ${ }^{3}$, Clairton Marcolongo-Pereira ${ }^{4}$, \\ Mauro Pereira Soares ${ }^{5}$, Eliza S.V. Sallis ${ }^{5}$, Silvia R.L. Ladeira ${ }^{5}$ e Ana Lucia Schild ${ }^{5 *}$
}

\begin{abstract}
Estima-Silva P., Molarinho K.R., Marcolongo-Pereira C., Soares M.P., Sallis E.S.V., Ladeira S.R.L. \& Schild A.L. 2016. [Sudden death in cattle in southern Brazil: epidemiology and diagnosis.] Morte súbita em bovinos no Sul do Rio Grande do Sul: epidemiologia e diagnóstico. Pesquisa Veterinária Brasileira 36(1):19-23. Laboratório Regional de Diagnóstico, Faculdade de Veterinária, Universidade Federal de Pelotas, Campus Universitário s/n, Pelotas, RS 96010-900, Brazil. E-mail: alschild@terra.com.br

A survey of cases with history of sudden death in cattle was conducted in all necropsy protocols of the Regional Diagnostic Laboratory of the Veterinary School of the Federal University of Pelotas (LRD/UFPel) from 2000 to 2014. We considered animals that had died unexpectedly without prior illness or clinical signs 24 hours before death (Category 1), or before they were moved or suffered some type of management and died after falls or trembling (Category 2). We identified 72 cases (3.5\%) from those two categories of a total of 2,031 materials of cattle received in LRD/UFPel. The cases occurred in all seasons of the year. In 34 cases $(47.2 \%)$, affected cattle had more than four years of age, in 23 cases (31.9\%) cattle had between two and three years, and in 11 cases (15.3\%) they were up to one year of age. In four protocols (5.6\%) age was not reported. In 62 cases (86.1\%) the cattle were in an extensive grazing system, in seven (9.7\%), they were in a semi-intensive grazing system, and in three cases (4.2\%), they were maintained in an intensive farming system. Out of the 72 cases observed, $52(72.2 \%)$ were classified in Category 1 and 20 (27.8\%) in Category 2. The diseases that caused more often sudden death, were cerebral babesiosis $(10 / 72)$, organophosphates poisoning (10/72), anthrax (7/72), bacillary hemoglobinuria (5/72), and fulguration (3/72). Out of the 18 cases considered inconclusive, just five full necropsiey were performed, and of the other 15, the material submitted was considered negative for Bacillus anthracis, and the material did not allow investigation for other diseases. The results showed that sudden deaths in southern Rio Grande do Sul are caused by diseases, mostly well-known and endemic to the region, which can be controlled or prevented by vaccination and appropriate management. Organs sent to the lab instead of the full body, limit the possibilities of conclusive diagnosis in cases of sudden death. The large number of negative cases regarding Bacillus anthracis infection was due to anthrax being a frequent suspicion when cattle appear dead without clinical signs, and the result of sending inappropriate material for the right diagnosis.
\end{abstract}

INDEX TERMS: Sudden death, epidemiology, cattle.

${ }^{1}$ Recebido em 18 de maio de 2015.

Aceito para publicação em 26 de novembro de 2015.

${ }^{2}$ Programa de Pós-Graduação em Veterinária, Faculdade de Veterinária (FV), Universidade Federal de Pelotas (UFPel), Campus Universitário, Pelotas, RS 96010-610, Brasil.

${ }^{3}$ Graduanda em Veterinária, bolsista de IC/CNPq, FV-UFPel, Campus Universitário, Pelotas, RS 96010-610, Brasil.

${ }^{4}$ Faculdade de Veterinária, Centro Universitário Ritter dos Reis (UniRitter), Rua Orfanotrofio 555, Alto Teresópolis, Porto Alegre, RS 90840-440, Brasil.

${ }^{5}$ Laboratório Regional de Diagnóstico, FV-UFPel, Campus Universitário, Pelotas, RS 96010-610.*Autor para correspondência: alschild@terra.com.br
RESUMO.- Foi realizado um levantamento nos protocolos de necropsias de bovinos com histórico de morte súbita ou superaguda recebidos no Laboratório Regional de Diagnóstico da Faculdade de Veterinária da Universidade Federal de Pelotas (LRD/UFPel) entre 2000 e 2014. Para o estudo foram considerados os casos em que os animais tinham morrido inesperadamente sem apresentação de uma doença prévia ou sinal clínico no intervalo de 24 horas antes da observação do cadáver (Categoria 1) ou bovinos movimentados ou que sofreram algum tipo de manejo e morreram 
após quedas ou tremores com observação destes sinais (Categoria 2). Foram identificados 72 casos ou surtos incluídos nestas duas categorias de um total de 2.031 cadáveres/ materiais de bovinos recebidos no LRD/UFPel no período, representando $3,5 \%$ do total. Os casos ocorreram em todas as épocas do ano e em 34 casos $(47,2 \%)$ os bovinos afetados eram adultos, em 23 casos $(31,9 \%)$ tinham entre dois e três anos e em $11(15,3 \%)$ tinham até um ano de idade. Em quatro protocolos $(5,6 \%)$ a idade não foi informada. Em 62 casos $(86,1 \%)$ a forma de criação era extensiva, em sete $(9,7 \%)$ a forma era semi-intensiva e em três $(4,2 \%)$ a forma era intensiva. Dos 72 casos/surtos observados 52 (72,2\%) foram classificados na Categoria 1 e $20(27,8 \%)$ na Categoria 2 . As enfermidades que mais causaram morte súbita ou superaguda foram: babesiose cerebral (10/72), intoxicação por organofosforados (10/72), carbúnculo hemático (7/72), hemoglobinúria bacilar (5/72) e fulguração $(3 / 72)$. Dos 18 casos inconclusivos em apenas cinco foi realizada a necropsia completa e nos 15 casos negativos a Bacillus anthracis o material remetido não permitiu a pesquisa de outras enfermidades. Os resultados obtidos, permitiram concluir que as mortes súbitas na região Sul do Rio Grande do Sul são causadas por doenças, na sua grande maioria, bem conhecidas e endêmicas da região e que podem ser controladas ou evitadas por vacinação e manejo adequados. 0 envio de órgãos ao laboratório, quando não é possível enviar o cadáver completo, pode limitar o diagnóstico conclusivo em casos de morte súbita/ superaguda. 0 número expressivo de casos negativos a Bacillus anthracis ocorre em consequência de carbúnculo hemático ser uma suspeita frequente quando bovinos aparecem mortos sem a apresentação de sinais clínicos resultando no envio de material inadequado para a realização de outros diagnósticos.

TERMOS DE INDEXAÇÃO: Morte súbita, morte superaguda, epidemiologia, bovinos.

\section{INTRODUÇÃO}

A expressão morte súbita é definida como a morte que ocorre inesperadamente em geral sem a apresentação de sinais clínicos por animais que são observados com relativa frequência (Radostits et al. 2007). A definição do termo é muitas vezes ambígua, dependendo da filosofia individual do avaliador e é mencionada em casos de bovinos confinados que morrem inesperadamente. A morte súbita pode ser definida como a morte clinicamente inexplicável, que ocorre durante uma atividade normal, em um período entre 12 e 24 horas (Borges et al. 2015).

0 emprego da expressão morte súbita, para designar mortalidade de bovinos que não apresentam sinais clínicos de doença prévia e são encontrados mortos de um dia para o outro, é frequente na região Sul do Rio Grande do Sul. Muitas vezes essas mortes são atribuídas a doenças desconhecidas ou misteriosas causadas por agentes até então não descritos e isso traz uma grande preocupação para produtores e trabalhadores rurais que imaginam ver o gado dizimado em poucos dias em consequência das mesmas. Na maioria das vezes, essas enfermidades são de curso superagudo, ou seja, os sinais clínicos são rápidos e muitas vezes sutis e podem passar despercebidos ou mesmo não serem observados induzindo à falsa ideia de que os animais morreram sem apresentação de sinais.

No Brasil, morte súbita associada ao exercício ocorre em bovinos pela ingestão de plantas do gênero Palicourea, Amorimia, Arrabidea e Pseudocalymma. Em geral bovinos intoxicados, ao serem movimentados, sofrem quedas repentinas com tremores musculares e a morte ocorre em poucos minutos (Tokarnia et al. 2012).

No Rio Grande do Sul morte súbita associada ao exercício tem sido observada em bovinos na região norte do Estado devido ao consumo de Amorimia exotropica (Pavarini et al. 2011). Na região Sul do Rio Grande do Sul surtos de morte súbita foram atribuídos, também, à carência de cobre, no entanto nestes casos não há relação direta com o exercício (Riet-Correa et al. 1993, Marques et al. 2003). A intoxicação por organofosforados causa também morte súbita em ruminantes e tem sido diagnosticada pelo uso inadequado destes produtos (Grecco et al. 2009, Santos et al. 2014). Na região de fronteira com o Uruguai ocorrem casos de morte súbita por carbúnculo hemático (Schild et al. 2006).

Os objetivos deste trabalho foram: relatar e estabelecer as diferenças entre surtos ou casos individuais de mortes por enfermidades de evolução superaguda ou morte súbita não associadas ao exercício que ocorreram em bovinos entre 2000 e 2014 na região Sul do Rio Grande do Sul; discutir as diferentes causas destas mortes e sua epidemiologia, estabelecendo o diagnóstico diferencial entre as mesmas; e fornecer subsídios a veterinários de campo para os possíveis diagnósticos.

\section{MATERIAL E MÉTODOS}

Foi realizado um levantamento nos protocolos de necropsias de bovinos com histórico de morte súbita ou superaguda recebidos no Laboratório Regional de Diagnóstico da Faculdade de Veterinária da Universidade Federal de Pelotas (LRD/UFPel) entre 2000 e 2014. Foram resgatados os dados epidemiológicos referentes à idade, a forma de criação e a procedência de cada bovino. Considerou-se apenas os casos em que no protocolo estava claramente definido que os bovinos tinham morrido inesperadamente sem apresentação de uma doença prévia ou qualquer sinal clínico no intervalo máximo de 24 horas antes da observação do cadáver (Categoria 1) ou bovinos movimentados ou que sofreram algum tipo de manejo e morreram em poucos minutos após quedas ou tremores com observação destes sinais (Categoria 2).

\section{RESULTADOS}

Foram identificados 72 casos individuais ou surtos de morte súbita em bovinos de um total de 2.031 cadáveres/materiais desta espécie recebidos no LRD/UFPel entre 2000 e 2014 , representando $3,5 \%$ do total de casos analisados no período. Os diagnósticos dos casos/surtos são apresentados no Quadro 1. Em 18 oportunidades (25\%) o diagnóstico foi inconclusivo e em 15 (20,8\%) o diagnóstico foi negativo a Bacillus anthracis.

Os casos ocorreram em todas as épocas do ano e em 34 casos $(47,2 \%)$ os bovinos afetados eram adultos, em 23 casos $(31,9 \%)$ tinham entre dois e três anos e em 11 $(15,3 \%)$ tinham até um ano de idade. Em quatro protocolos $(5,6 \%)$ a idade não foi informada. Em 62 casos $(86,1 \%)$ 
Quadro 1. Diagnóstico, no de surtos, época de ocorrência, variação da mortalidade nos surtos e categoria de morte súbita/superaguda em bovinos no Sul do Rio Grande do Sul

\begin{tabular}{|c|c|c|c|c|c|c|c|c|}
\hline \multirow[t]{2}{*}{ Diagnóstico } & \multirow{2}{*}{$\begin{array}{l}\text { № de } \\
\text { surtos }\end{array}$} & \multicolumn{4}{|c|}{ Época do Ano ${ }^{a}$} & \multirow{2}{*}{$\begin{array}{c}\text { Variação da } \\
\text { Mortalidade (\%) }\end{array}$} & \multicolumn{2}{|c|}{ Categorias $^{\mathrm{b}}$} \\
\hline & & $\mathrm{P}$ & V & 0 & I & & 1 & 2 \\
\hline Babesiose cerebral $^{c}$ & 10 & 1 & 4 & 5 & - & $0,2-8,4$ & 10 & - \\
\hline Intoxicação por ORFs ${ }^{\mathrm{d}, \mathrm{e}}$ & 10 & 3 & 7 & - & - & $1,8-87$ & 1 & 9 \\
\hline Carbúnculo hemático & 7 & 2 & 3 & 2 & - & $0,8-25$ & 5 & 2 \\
\hline Hemoglobinúria bacilar & 5 & - & 1 & - & 4 & $0,3-2,2$ & 5 & - \\
\hline Fulguraçãof & 3 & 1 & - & 1 & 1 & 25 & 3 & - \\
\hline Intoxicação por Xanthium spp. & 1 & 1 & - & - & - & 7,1 & 1 & - \\
\hline Intoxicação por Perreiya flavipes & 1 & - & - & - & 1 & 6,6 & 1 & - \\
\hline Intoxicação por Prunus selowii & 1 & - & - & 1 & - & 16,7 & - & 1 \\
\hline Carência de Cobre & 1 & - & - & - & 1 & 5,6 & - & 1 \\
\hline Negativo a Bacillus anthracis & 15 & 5 & 1 & 7 & 2 & $0,1-25$ & 15 & - \\
\hline Inconclusivos & 18 & 3 & 4 & 5 & 6 & $0,1-20$ & 11 & 7 \\
\hline TOTAL & 72 & 16 & 20 & 21 & 15 & & 52 & 20 \\
\hline
\end{tabular}

\footnotetext{
${ }^{\mathrm{a}} \mathrm{P}=$ primavera, $\mathrm{V}=$ verão, $\mathrm{O}=$ outono, $\mathrm{I}=$ inverno; ${ }^{\mathrm{b}}$ Categoria $1=$ animais que aparecem mortos sem sinais clínicos prévios em até 24 horas após a última observação, Categoria $2=$ morte durante ou logo após manejo com observação de quedas e tremores; ${ }^{c}$ em três surtos nos protocolos de necropsia não estava especificado o número total de animais sob risco; ${ }^{\mathrm{d}}$ ORFs = organofosforados; ${ }^{\mathrm{e}}$ em seis surtos no protocolo de necropsia não estava especificado o número total de animais sob risco (Santos et al. 2014); ${ }^{\mathrm{f}}$ em dois surtos nos protocolos de necropsia não estava especificado o número total de animais sob risco.
}

a forma de criação era extensiva, em sete $(9,7 \%)$ a forma era semi-intensiva e em três $(4,2 \%)$ a forma era intensiva. Em relação a procedência dos casos/surtos, $22(30,6 \%)$ eram provenientes de Santa Vitória do Palmar, 11 (15,3\%) eram do Capão do Leão e sete $(9,7 \%)$ eram de Pelotas. Dom Pedrito, Rio Grande e São Lourenço do Sul tiveram cinco casos $(6,9 \%)$ cada e três casos $(4,2 \%)$ eram provenientes de Piratini. Arroio Grande, Canguçu e Pedras Altas tiveram dois casos $(2,8 \%)$ cada e Aceguá, Bagé, Caçapava, Camaquã, Herval, Jaguarão, Pedro Osório e Turuçu tiveram um caso $(1,4 \%)$ cada. Dos 72 casos/surtos observados 52 (72,2\%) foram classificados na Categoria 1 e $20(27,8 \%)$ na Categoria 2. Os dados epidemiológicos estão sumarizados no Quadro 1. No surto diagnosticado como intoxicação por cobre a dosagem do microelemento variou de 3,7 a 3,9 ppm no fígado de três bovinos mortos (Marques et al. 2003).

\section{DISCUSSÃO}

0 presente trabalho permitiu identificar as principais enfermidades diagnosticadas em bovinos com histórico de morte súbita necropsiados no laboratório ou a campo, na região Sul do Rio Grande do Sul. Ficou evidenciado que a principal categoria de bovinos com esse histórico é a de animais adultos e acima de dois anos de idade criados extensivamente. A condição tem sido relatada como frequente em bezerros e atribuída a falha cardíaca, porém sem a observação de lesões evidentes no miocárdio que confirmassem a hipótese (Bradley et al. 1981). Considerando os diagnósticos conclusivos (39/72), as principais causas de morte súbita em bovinos foram as provocadas por doenças parasitárias e infecciosas, com $56,4 \%$ dos diagnósticos. Em segundo lugar foram as causas tóxicas com $33,3 \%$ dos diagnósticos e as demais (metabólicas e ambientais) com 10,3\% dos casos. Muitas das enfermidades apontadas como causa de morte súbita descritas neste trabalho têm sido relatadas em outros países causando a mesma condição (Borges et al.
2015). Por outro lado, diferenças epidemiológicas demonstram que muitas enfermidades que cursam com sinais clínicos superagudos e causam morte súbita em algumas regiões não o fazem em outras.

Os diagnósticos conclusivos mais frequentes foram a babesiose cerebral e a intoxicação por organofosforados (ORFs). Na morte súbita por babesiose cerebral todos os casos foram enquadrados na Categoria 1. A intensidade dos sinais clínicos na babesiose cerebral depende de vários fatores entre eles a virulência da cepa e a quantidade de inóculo, os quais tornam o quadro clínico mais grave (Farias 2007) e a morte ocorre rapidamente, muitas vezes, sem que este quadro clínico seja observado. Por outro lado, é importante ressaltar que a babesiose cerebral pode ocorrer com evolução de até 4 dias ou mais, principalmente em animais jovens, que são mais resistentes (Farias 2007). Nos surtos aqui relatados os animais eram adultos em $50 \%$ dos casos e tinham entre dois e três anos de idade em $20 \%$.

Carbúnculo hemático foi observado em sete oportunidades das quais em cinco $(71,4 \%)$ a classificação foi na Categoria 1 e em duas $(28,6 \%)$ a classificação foi na Categoria 2. Esta é uma enfermidade endêmica e de evolução superaguda que ocorre na região sul do Rio Grande do Sul, principalmente nos municípios de fronteira com o Uruguai e Argentina (Schild et al. 2006), sendo que, via de regra, nesta região os rebanhos são vacinados anualmente. Cinco dos sete surtos diagnosticados ocorreram no ano 2006 quando houve um aumento na incidência da doença devido a não vacinação dos bovinos em diversos municípios da região (Schild et al. 2006). Isto justifica o fato de que os casos suspeitos que resultam negativos são mais numerosos que os casos positivos da doença.

Hemoglobinúria bacilar (HB) é uma clostridiose endêmica da região sul do Rio Grande do Sul e foi causa de morte súbita em cinco oportunidades, sendo em todos os casos classificados na Categoria 1. Esta enfermidade quando os animais 
aparecem mortos de um dia para o outro é confundida com carbúnculo hemático e por esta razão a necropsia não é realizada determinando uma frequência provavelmente subestimada. Por outro lado, a vacinação contra as clostridioses, incluindo a HB, é realizada sistematicamente na região o que pode, também, levar a diminuição da frequência da doença.

Em nove dos 10 casos de intoxicação por ORFs os bovinos foram incluídos na Categoria 2, sugerindo que nestes casos a utilização destes produtos pouco antes do início das mortes deve ser sempre investigada. Estes sinais têm sido frequentemente descritos na intoxicação por estes produtos em ruminantes na região do estudo (Grecco et al. 2009, Santos et al. 2014). Apesar de que podem morrer animais após um período de tempo mais prolongado devido a diferenças de dose e susceptibilidade, os sinais clássicos dessa intoxicação são facilmente detectáveis.

Intoxicações que causam lesão hepática aguda como as intoxicações por Perreyia flavipes (Soares et al. 2008), Xanthium spp. (Driemeier et al. 1999) e intoxicação por Prunus selowii (Gava et al. 1992), são pouco frequentes e o diagnóstico nos casos relatados neste trabalho foi realizado pelas lesões macroscópicas e histológicas além da epidemiologia e pela presença dos respectivos agentes tóxicos.

No presente estudo é importante destacar que dos 18 casos com diagnóstico inconclusivo, apenas em cinco havia sido realizada a necropsia completa dos animais por técnicos do LRD no próprio laboratório ou a campo. 0 envio de órgãos em gelo ou formalina muitas vezes é necessário para diminuir o risco de autólise do cadáver em função da distância entre a propriedade e o laboratório. Por outro lado, frequentemente, isto impede ou dificulta a realização do diagnóstico conclusivo muitas vezes devido a erros de interpretação de lesões observadas durante a necropsia ou encaminhamento de material inadequado para o diagnóstico. Além disso, devem ser considerados também, junto com os casos inconclusivos resultante de órgãos recebidos, os casos suspeitos de carbúnculo hemático que resultaram negativos (negativo à Bacillus anthracis). Deve salientar-se que na suspeita desta enfermidade a necropsia não é recomendada e o material enviado (canela, orelha ou sangue refrigerados) são insuficientes ou inadequados para a realização de outros diagnósticos. A realização da necropsia após o exame direto ser negativo provavelmente aumentaria a eficiência do diagnóstico nestes casos. A tendência de considerar carbúnculo hemático como a única causa de morte sem apresentação de sinais clínicos em bovinos é frequente, principalmente na região Sul do Estado o que acaba, também, por elevar o número de casos negativos. A epidemiologia da doença, que é bastante característica, com ocorrência de mortes pingadas em verões secos com intervalos de uma semana ou mais, deve ser considerada para o diagnóstico (Schild et al. 2006).

Com relação a procedência dos bovinos que tiveram morte súbita houve um percentual expressivo $(30,6 \%)$ de casos provenientes do município de Santa Vitória do Palmar. Neste mesmo município a babesiose não ocorre devido à ausência do carrapato Rhipicephalus microplus e apenas um surto em consequência da introdução acidental do transmissor da enfermidade foi diagnosticado em 2008, mas neste caso a mesma não cursou com morte súbita (Schild et al. 2008). Quatro dos cinco casos de HB ocorreram neste município já que a doença é endêmica na região e frequentemente cursa com morte súbita, sendo necessária a vacinação sistemática anual dos bovinos. Cabe destacar, ainda, que seis casos suspeitos de carbúnculo hemático provenientes de Santa Vitória do Palmar resultaram negativos no presente estudo e não puderam ser testados para HB já que as necropsias não foram realizadas. Os sete casos de intoxicação por ORFs observados neste município foram diagnosticados entre 2013 e 2014, sendo todos consequência do uso inadequado do produto (Santos et al. 2014). Estes casos de morte súbita podem ser evitados com o uso dos produtos nas diluições e doses recomendadas pelos fabricantes (Santos et al. 2014).

Os bovinos criados extensivamente foram mais frequentemente afetados por morte súbita, chegando a $86,1 \%$ dos casos. Essa maior incidência deve-se ao fato de que a região Sul do Rio Grande do Sul é uma área de grandes extensões de terra com propriedades rurais que criam bovinos a pasto frequentemente em áreas de restevas de arroz ou soja. Em consequência disso, muitos casos de morte súbita são atribuídos, também, ao envenenamento por agrotóxicos, o que não tem sido comprovado na região.

No Brasil os surtos de morte súbita associados ao exercício ocorrem em consequência da ingestão de plantas tóxicas, muitas das quais têm sido largamente estudadas como as intoxicações por diversas espécies de Palicourea e Amorimia. (Pavarini et al. 2011 Schons et al. 2011, Tokarnia et al. 2012). No Sul do Rio Grande do Sul, região onde este estudo foi realizado, no entanto, nenhuma destas plantas têm sido encontradas.

\section{CONCLUSÕES}

Com base nos resultados deste trabalho, é possível desmistificar a afirmativa de que as mortes súbitas ou superagudas na região Sul do Estado são causadas por agentes não identificados, já que ficou claramente definido que as doenças que as causam, na sua grande maioria, são bem conhecidas, endêmicas da região e podem ser controladas ou evitadas.

Foi possível concluir, também, que a remessa de órgãos ao laboratório, apesar de ser uma prática importante para realização de vários diagnósticos em bovinos, quando é impossível a remessa do cadáver completo, pode limitar a realização do diagnóstico conclusivo.

O elevado número de casos negativos a Bacillus anthracis deve-se, que carbúnculo hemático é uma enfermidade que restringe a realização da necropsia e o material remetido ao laboratório é inadequado para a realização de outros diagnósticos.

Concluiu-se ainda, que os casos mais frequentes de morte súbita ou superaguda ocorrem em bovinos adultos criados extensivamente que aparecem mortos sem observação de sinais clínicos em um período máximo de 24 horas.

\section{REFERÊNCIAS}

Bradley R., Markson L.M. \& Bailey J. 1981, Sudden death and myocardial necrosis in cattle. J. Pathol. 135:19-38. 
Borges J.R.J., Casteel S.W. \& Schaeffer J.W. 2015. Collapse and sudden death, p.214-220. In: Smith B.P. (Ed.), Large Animal Internal Medicine. 5th ed. Mosby, St Louis. 1661p.

Driemeier D., Irigoyen L.F., Loretti A.P., Colodel E.M. \& Barros C.S.L. 1999. Intoxicação espontânea pelos frutos de Xanthium cavanillesii (Asteraceae). em bovinos no Rio Grande do Sul. Pesq. Vet. Bras. 19(1):12-18.

Farias N.A. 2007. Tristeza parasitária bovina, p.524-532. In: Riet-Correa F., Schild A.L., Lemos R.A.A. \& Borges J.R.J. (Eds), Doenças de Ruminantes e Equídeos. Vol.1. 3aㅡ ed. Pallotti, Santa Maria, RS.

Gava A., Stolf L., Neves D.S., Stolf O., Varaschin M.S. \& Ferreira E.M.M. 1992. Intoxicação experimental por Prunus selowii em bovinos. Pesq. Vet. Bras. 12:1-4.

Grecco F.B., Schild A.L., Soares M.P., Rafii M.B., Sallis E.S.V. \& Damé M.C. 2009. Intoxicação por organofosforados em búfalos (Bubalus bubalis) no Rio Grande do Sul. Pesq. Vet. Bras. 29(3):211-214.

Marques A.P., Riet-Correa F., Soares M.P., Ortolani E.L. \& Giuliodori M.J. 2003. Mortes súbitas em bovinos associadas à carência de cobre. Pesq. Vet. Bras. 23(1):21-32.

Pavarini S.P., Soares M.P., Bandarra P.M., Gomes D.C., Bandinelli M.B., Cruz C.E.F. \& Driemeier D. 2011. Mortes súbitas causadas por Amorimia exotropica (Malpighiaceae) no Rio Grande do Sul. Pesq. Vet. Bras. 31:291296.

Radostits O.M., Gay C.C., Hinchcliff K.W. \& Constable P.D. 2007. Veterinary medicine: a textbook of the diseases of cattle, horses, sheep, pigs, and goats. 10th ed. W.B. Saunders, Philadelphia. 2156p.

Riet-Correa F., Bondan E.F., Mendez M.C., Moraes S.S. \& Concepción M.R. 1993. Efeito da suplementação com cobre e doenças associadas à carência de cobre em bovinos no Rio Grande do Sul. Pesq. Vet. Bras. 13(3/4):45-49.

Santos B.L., Marcolongo-Pereira C., Stigger A.L., Coelho A.C.B., Soares M.P., Sallis E.S.V., Barreto F. \& Schild A.L. 2014. Uso inadequado de organofosforados: uma prática de risco para bovinos no Sul do Rio Grande do Sul. Pesq. Vet. Bras. 34(7):655-658.

Schild A.L., Sallis E.S.V., Soares M.P., Ladeira S.R.L., Schram R., Priebe A.P., Almeida M.B. \& Riet-Correa F. 2006. Anthrax in cattle in southern Brazil: 1978-2006. Pesq. Vet. Bras. 26(4):243-248.

Schild A.L., Ruas J. L., Farias N.A., Grecco F.B. \& Soares M.P. 2008. Aspectos epidemiológicos de um surto de babesiose cerebral em zona livre de carrapato. Ciência Rural 38(9):2646-2649.

Schons S.V., Mello T. L., Riet-Correa F. \& Schild A. L. 2011. Poisoning by Mascagnia (Amorimia) sepium in sheep in northern Brazil. Toxicon 57:781-786.

Soares M.P., Quevedo P.S. \& Schild A.L. 2008. Intoxicação por larvas de Perreyia flavipes em bovinos na região sul do Rio Grande do Sul. Pesq. Vet. Bras. 28(3):169-173.

Tokarnia C.H., Brito M.F., Barbosa J.D., Peixoto P.V \& Döbereiner J. 2012. Plantas Tóxicas do Brasil. Helianthus, Rio de Janeiro, p.29-70. 